\title{
OSCILLATION CRITERIA OF SECOND-ORDER NON-LINEAR DYNAMIC EQUATIONS WITH INTEGRO FORCING TERM ON TIME SCALES
}

\author{
S.S. Negi, School of Basic Sciences, Indian Institute of Technology Mandi, India, \\ sssshekhar.singh17@gmail.com, \\ S. Abbas, School of Basic Sciences, Indian Institute of Technology Mandi, India, \\ sabbas.iitk@gmail.com,
}

M. Malik, School of Basic Sciences, Indian Institute of Technology Mandi, India, malikiisc@gmail.com

\begin{abstract}
This paper is concerned with the oscillatory properties of second order non-linear dynamic equation with integro forcing term on an arbitrary time scales. We reduce our original dynamic equation into an alternate equation by introducing a function of forward jump operator. To study oscillations we establish some crucial Lemmas and employ generalized Riccati transformation technique which transforms our second order dynamic equation into the first order dynamic equation on an arbitrary time scales. These results also guarantee that the solution of our equation oscillates. Furthermore, we establish the Kamenev-type oscillation criteria of our system. At the end, we consider a second order dynamic equation on time scales with deviating argument and compare it with our result which gives the sufficient conditions of oscillation of it.
\end{abstract}

Keywords: time scale; dynamic equation; Riccati transformation technique; oscillation.

\section{Introduction and Preliminaries}

The oscillation theory of differential equation and difference equation has been receiving a lot of attention in the past few decades. It is a very important concept in the qualitative behaviour of the solutions of both types of equations. Many researchers have taken an interest in studying the oscillation and non-oscillation criteria of the solution of both differential and difference equations [1-3]. The problem of obtaining the sufficient conditions for oscillation of both types of equations takes much time. Therefore, it is necessary to find a single way for both equations.

The time scale theory which removes this ambiguity has been first introduced by a German mathematician Stefan Hilger in his Ph.D. dissertation (1988) [4]. The significance of this theory is that it does not only avoid the dual analysis but also harmonize both continuous and discrete calculus.

Time scale is a non-empty closed subset of the real numbers (i.e., $\mathbb{R}$ ), e.g., set of natural numbers $(\mathbb{N})$, integers $(\mathbb{Z})$, Cantor set, set of harmonic numbers etc. In this way, the results do not only relate to the set of real numbers or set of integers but also pertain to more general times scales. The three most popular examples of calculus on time scales are differential calculus $(\mathbb{T}=\mathbb{R})$, difference calculus $(\mathbb{T}=\mathbb{Z})$, and quantum calculus $\left(\mathbb{T}=q^{\mathbb{Z}} \bigcup\{0\}, q>1\right)$, for more details see $[5,6]$ and references therein. It has many applications in various fields, e.g., population dynamics, economics, neural networks, quantum physics and social science etc. $[7,8]$.

We briefly recall some basic definitions, useful Theorems, Lemmas, assumptions and basic facts of time scales etc. 
Definition 1. $[5,6]$ For $t \in \mathbb{T}$, we define a forward jump operator $\sigma: \mathbb{T} \rightarrow \mathbb{T}$ by $\sigma(t):=\inf \{s \in \mathbb{T}: s>t\}$, if $t<\sigma(t)$ and $t<\sup \mathbb{T}$ as well as $t=\sigma(t)$, then $t$ is right-scattered and right dense respectively. A backward jump operator $\rho: \mathbb{T} \rightarrow \mathbb{T}$ by $\rho(t):=\sup \{s \in \mathbb{T}: s<t\}$, if $t>\rho(t)$ and $t>\inf \mathbb{T}$ as well as $t=\rho(t)$, then $t$ is left-scattered and left dense respectively. The graininess operator $\mu: \mathbb{T} \rightarrow[0, \infty)$ is defined by $\mu(t)=\sigma(t)-t$.

Remark 1. We put inf $\phi=\sup \mathbb{T}$ (i.e., $\sigma(t)=t$ if $\mathbb{T}$ has a maximum $t$ ), $\sup \phi=\inf \mathbb{T}$ (i.e., $\rho(t)=t$ if $\mathbb{T}$ has a minimum $t$ ).

Definition 2. [5,6] A function $f: \mathbb{T} \rightarrow \mathbb{R}$ is called $r d$-continuous provided it is continuous at all right-dense points in $\mathbb{T}$ and its left-sided limit exists (finite) at left-dense points in $\mathbb{T}$, denoted by $C_{r d}=C_{r d}(\mathbb{T})=C_{r d}(\mathbb{T}, \mathbb{R})$. We define some notations as follows:

$+\mathbb{C}_{r d}^{\Delta}(\mathbb{T})=\left\{q: q(t)\right.$ is positive rd-continuous function and $\left.q^{\Delta}(t) \in \mathbb{C}_{r d}(\mathbb{T})\right\}$.

$+\mathbb{C}_{r d}^{\Delta+}(\mathbb{T})=\left\{q: q(t), q^{\Delta}(t)\right.$ are positive rd-continuous functions $\}$.

Definition 3. [5,6] A function $G: \mathbb{T} \rightarrow \mathbb{R}$ is called an anti-derivative of $g: \mathbb{T} \rightarrow \mathbb{R}$, provided $G^{\Delta}(t)=g(t) \forall t \in \mathbb{T}$. Then $\forall a, b \in \mathbb{T}$, the Cauchy integral is defined by

$$
\int_{a}^{b} g(s) \Delta(s)=G(b)-G(a) \text {. }
$$

Define

$$
\mathbb{T}^{\kappa}= \begin{cases}\mathbb{T}-\{l\}, & \text { if } \mathbb{T} \text { has a left-scattered maximum } l, \\ \mathbb{T}^{\kappa}=\mathbb{T}, & \text { otherwise. }\end{cases}
$$

Definition 4. [5,6] For a function $f: \mathbb{T} \rightarrow \mathbb{R}$ and $t \in \mathbb{T}^{\kappa}$, we define $f^{\Delta}(t)$, to be a number (provided it exists) with the property that given any $\epsilon>0$, there exists a neighborhood $\mathcal{A}=(t-\delta, t+\delta) \bigcap \mathbb{T}$ for some $\delta>0$ such that

$$
\left|[f(\sigma(t))-f(r)]-f^{\Delta}(t)[\sigma(t)-r]\right| \leq \epsilon|\sigma(t)-r| \quad \forall r \in \mathcal{A}
$$

Thus, we call $f^{\Delta}(t)$ the delta or Hilger derivative of $f$ at $t$. $f$ is also called differentiable at $t$.

Theorem 1. [5,6] For the functions $g, f: \mathbb{T} \rightarrow \mathbb{R}$ and $t \in \mathbb{T}^{\kappa}$, we have the following:

1. If $f$ is differentiable at $t$, then $f$ is continuous at $t$;

2. If $f$ is continuous at $t$ and $t$ is right-scattered, then $f$ has a delta derivative at $t$ and

$$
f^{\Delta}(t)=\frac{f(\sigma(t))-f(t)}{\mu(t)}
$$

3. If $t$ is right-dense, then $f$ is differentiable at $t$ if the limit

$$
f^{\Delta}(t)=\lim _{s \rightarrow t} \frac{f(t)-f(s)}{t-s}
$$

exists and has a finite value;

4. If $f$ is differentiable at $t$, then

$$
f^{\sigma}=f(\sigma(t))=f(t)+\mu(t) f^{\Delta}(t)
$$


5. If $f$ and $g$ both are differentiable at $t$, then the product $f g: \mathbb{T} \rightarrow \mathbb{R}$ is differentiable at $t$ and

$$
(f g)^{\Delta}(t)=f^{\Delta}(t) g(t)+f(\sigma(t)) g^{\Delta}(t)=f(t) g^{\Delta}(t)+f^{\Delta}(t) g(\sigma(t))
$$

6. If $g(t) g(\sigma(t)) \neq 0$ with $g(t) \neq 0$, then $\frac{f(t)}{g(t)}$ is differentiable at $t$ and

$$
\left(\frac{f}{g}\right)^{\Delta}(t)=\frac{f^{\Delta}(t) g(t)-f(t) g^{\Delta}(t)}{g(t) g(\sigma(t))} .
$$

Definition 5. $[5,6]$ A function $q: \mathbb{T} \rightarrow \mathbb{R}$ is called regressive if $1+\mu(t) q(t) \neq 0, \forall t \in \mathbb{T}$. We denote the collections of all functions $h: \mathbb{T} \rightarrow \mathbb{R}$ which are rd-continuous and regressive by $\mathcal{R}$ and $\mathcal{R}^{+}=\{q \in \mathcal{R}: 1+\mu(t) p(t)>0$ for all $t \in \mathbb{T}\}$.

Definition 6. [5,6] (Time scale version of exponential function). If $q \in \mathcal{R}$, then we define the exponential function by

$$
e_{q}(t, s)=\exp \left(\int_{s}^{t} \eta_{\mu(\tau)}(q(\tau)) \Delta \tau\right), \quad \forall t \in \mathbb{T}, s \in \mathbb{T}^{\kappa},
$$

where $\eta_{h}(z)$ is the cylinder transformation, which is defined by

$$
\eta_{h}(z)=\left\{\begin{array}{cl}
\frac{\log (1+h z)}{h}, & \text { if } h \neq 0, \\
z, & \text { if } h=0 .
\end{array}\right.
$$

Definition 7. [5,6] If $q \in \mathcal{R}$, then the first order linear dynamic equation

$$
y^{\Delta}(t)=q(t) y(t)
$$

is called regressive.

Theorem 2. [5,6] Suppose that (1) is regressive and fix $t_{0} \in \mathbb{T}$. Then $e_{q}\left(., t_{0}\right)$ is a solution of the initial value problem

$$
y^{\Delta}(t)=q(t) y(t), \quad y\left(t_{0}\right)=1
$$

on $\mathbb{T}$.

Theorem 3. [5,6] If (1) is regressive, then $e_{q}\left(., t_{0}\right)$ is the only solution of (2).

Theorem 4. $[5,6]$ If $p, q \in \mathcal{R}$, then

1. $e_{0}(t, s) \equiv 1$ and $e_{p}(t, t) \equiv 1$;

2. $e_{p}(\sigma(t), s)=(1+\mu(t) p(t)) e_{p}(t, s)$;

3. $e_{\ominus p}(t, s)=\frac{1}{e_{p}(t, s)}$;

4. $e_{p}(t, s) e_{q}(t, s)=e_{p \oplus q}(t, s)$; 
5. $\left(\frac{1}{e_{p}(., s)}\right)^{\Delta}=-\frac{p(t)}{e_{p}^{\sigma}(., s)}$

6. If $q \in \mathcal{R}^{+}$, then $e_{q}(t, s)>0$ for all $t \in \mathbb{T}$.

In this paper, we study the oscillation criteria of the second order non-linear dynamic equation with integro forcing term on time scale $\mathbb{T}$ :

$$
y^{\Delta \Delta}(t)+\beta y^{\Delta}(t)=B(t) y(t)+H^{\Delta}\left(t, y(t), \int_{-\infty}^{t} \mathcal{J}(t-s) \mathcal{H}(s, y(s)) \Delta s\right),
$$

where $B, \mathcal{J}: \mathbb{T} \rightarrow \mathbb{R}$ are the function of $t$, and the forcing terms $H: \mathbb{T} \times \mathbb{R}^{2} \rightarrow \mathbb{R}$ and $\mathcal{H}: \mathbb{T} \times \mathbb{R} \rightarrow \mathbb{R}$. For the alternate form of equation (3), we multiply (3) by a function $r^{\sigma}(t)$. We have

$$
r^{\sigma}(t) y^{\Delta \Delta}(t)+\beta r^{\sigma}(t) y^{\Delta}(t)=r^{\sigma}(t) B(t) y(t)+r^{\sigma}(t) H^{\Delta}\left(t, y(t), \int_{-\infty}^{t} \mathcal{J}(t-s) \mathcal{H}(s, y(s)) \Delta s\right) .
$$

From above relation, we obtain

$$
\begin{aligned}
& \underbrace{r^{\sigma}(t) y^{\Delta \Delta}(t)+r^{\Delta}(t) y^{\Delta}(t)}-r^{\Delta}(t) y^{\Delta}(t)+\beta r^{\sigma}(t) y^{\Delta}(t)= \\
& =r^{\sigma}(t) B(t) y(t)+r^{\sigma}(t) H^{\Delta}\left(t, y(t), \int_{-\infty}^{t} \mathcal{J}(t-s) \mathcal{H}(s, y(s)) \Delta s\right),
\end{aligned}
$$

which is equivalent to

$$
\begin{aligned}
& \left(r(t) y^{\Delta}(t)\right)^{\Delta}+\left(\beta r^{\sigma}(t)-r^{\Delta}(t)\right) y^{\Delta}(t)= \\
& =B(t) r^{\sigma}(t) y(t)+r^{\sigma}(t) H^{\Delta}\left(t, y(t), \int_{-\infty}^{t} \mathcal{J}(t-s) \mathcal{H}(s, y(s)) \Delta s\right) .
\end{aligned}
$$

We study oscillation criteria of (4). A non-trivial $y(t)$ such that $y(t) \in \mathbb{C}_{r d}^{\Delta}\left(\left[t_{y}, \infty\right) \mathbb{T}\right)$, $r(t) y^{\Delta}(t) \in \mathbb{C}_{r d}^{\Delta}\left(\left[t_{y}, \infty\right)_{\mathbb{T}}\right)$ for certain $t_{y} \geq t_{1}$ and satisfying (4) for $t_{y} \leq t$ is called nonoscillatory if it is eventually positive or eventually negative, otherwise it is called oscillatory. In other words, it is said to be oscillatory if it has an arbitrarily large number of zeros, i.e., there exists a sequence $\left\{s_{n}\right\}$ such that $\lim _{n \rightarrow \infty} s_{n}=\infty$ as well as $y\left(s_{n}\right)=0, \forall n$. A dynamic equation is called oscillatory if every solution is oscillatory, and non-oscillatory otherwise. Our attention is restricted to those solutions of (4) which exist on some $\left[t_{y}, \infty\right)_{\mathbb{T}}$ and satisfy $\sup \left\{|y(t)|: t>t_{*}\right\}>0$ for any $t_{y} \leq t_{*}$.

In the past few years, there have been many research activities concerning the oscillation of solutions of various forced second-order dynamic equations on time scales. The oscillation criteria of the second order linear and non-linear dynamic equations on time scales have been studied by many researchers, and we have plenty of essential papers, articles etc. [9-14]. In particular: In (2003), Erbe, et. al. [13] considered linear damped dynamic equation

$$
x^{\Delta \Delta}(t)+p(t) x^{\Delta}(t)+q(t) x(t)=0
$$

and non-linear equation

$$
x^{\Delta \Delta}(t)+p(t) x^{\Delta^{\sigma}}(t)+q(t)\left(f o x^{\sigma}\right)(t)=0
$$


to establish the oscillation criteria. In (2007), Saker, et. al. [14] gerneralized (5) as follows:

$$
\left(r(t) x^{\Delta}(t)\right)^{\Delta}+p(t) x^{\Delta^{\sigma}}(t)+q(t)\left(f \circ x^{\sigma}\right)(t)=0,
$$

and studied an oscillation criteria. Finally, in (2008), Erbe, et. al. [15] have considered a non-linear damped delay dynamic equation by introducing a constant term $\beta$, where $\beta$ is a quotient of odd positive integers and studied the sufficient conditions for oscillation:

$$
\left(r(t)\left(x^{\Delta}(t)\right)^{\beta}\right)^{\Delta}+p(t)\left(x^{\Delta^{\sigma}}(t)\right)^{\beta}+q(t) f(x(\tau(t)))=0,
$$

which extend and improve the results $[13,14]$.

In (2004), Bohner, et. al. [10] considered a second-order perturbed dynamic equation:

$$
\left(r(t)\left(x^{\Delta}(t)\right)^{\gamma}\right)^{\Delta}+F\left(t, x^{\sigma}(t)\right)=G\left(t, x(t), x^{\Delta}(t)\right)
$$

where $\gamma$ is a positive odd integer. In (2006), Agarwal, et. al. [9] modified equation (8)

$$
\left(r(t)\left(x^{\Delta}(t)\right)^{\gamma}\right)^{\Delta}+\underbrace{F(t, x(t))}=G\left(t, x(t), x^{\Delta}(t)\right)
$$

and both have established the sufficient conditions for oscillation. In (2010), Chen, et. al. [11] considered a dynamic equation with damping on time scale

$$
\left(\left(x^{\Delta}(t)\right)^{\gamma}\right)^{\Delta}+p(t)\left(x^{\Delta}(t)\right)^{\gamma}+q(t) f(x(\sigma(t))=0,
$$

and studied the oscillation criteria as well as established the Kamenev type and the Philostype oscillation criteria of it. Throughout this paper we denote $[a, \infty) \cap \mathbb{T}=[a, \infty)_{\mathbb{T}}$, where $\sup (\mathbb{T})=\infty$.

This paper is organized as follows: In this section, we establish some necessary Lemmas. In the next section, we use Riccati transformation technique to establish the sufficient conditions for oscillation of (4) and also establish the Kamenev type oscillation criteria. Finally, we consider another second order dynamic equation with deviating argument and establish the same for oscillation.

It will be convenient to make the following notations:

$$
\begin{aligned}
& Q(t)=\frac{\beta r^{\sigma}(t)-r^{\Delta}(t)}{r(t)}, \quad D\left(t, s^{*}\right)=e \frac{Q(t)}{1-\mu(t) Q(t)}\left(t, s^{*}\right), \quad M(t)=(p(t)-B(t)) r^{\sigma}(t), \\
& B_{1}(t)=\frac{\left(\delta^{\Delta}(t)-\delta^{\sigma}(t) Q(t) ð(t)\right)}{\delta(t)}, B_{2}(t)=\frac{\delta^{\sigma}(t) ð(t)}{\delta^{2}(t) r(t)}, \quad A_{2}(t)=\left(\frac{D^{\sigma}\left(t, s^{*}\right) \delta(t)}{r(t)\left(\delta^{\sigma}(t)\right)^{2} D\left(t, s^{*}\right)}\right), \\
& A_{1}=\left(\frac{\delta^{\Delta}(t)}{\delta^{\sigma}(t)}-\frac{D^{\sigma}\left(t, s^{*}\right) \delta(t)}{D\left(t, s^{*}\right) \delta^{\sigma}(t)} Q(t)\right) .
\end{aligned}
$$

To establish our results we use the following assumptions:

$\left(\mathbf{U}_{1}\right) B:\left[t_{1}, \infty\right)_{\mathbb{T}} \rightarrow \mathbb{R}$ is a negative rd-continuous function and $p:\left[t_{1}, \infty\right)_{\mathbb{T}} \rightarrow \mathbb{R}$ is a positive rd-continuous function;

$\left(\mathbf{U}_{\mathbf{2}}\right) B:\left[t_{1}, \infty\right)_{\mathbb{T}} \rightarrow \mathbb{R}$ is an rd-continuous function and $p:\left[t_{1}, \infty\right)_{\mathbb{T}} \rightarrow \mathbb{R}$ is a positive rd-continuous function such that $p(t)-B(t)>0$; 
$\left(\mathbf{U}_{3}\right) Q:\left[t_{1}, \infty\right)_{\mathbb{T}} \rightarrow \mathbb{R}$ is a positive rd-continuous function such that $1-\mu(t) Q(t)>0$, and $r(t) \in{ }^{+} C^{\Delta}{ }_{r d}(\mathbb{T})$;

$\left(\mathbf{U}_{4}\right) H: \mathbb{T} \times \mathbb{R}^{2}$ is a $\Delta$-derivative finction (w.r.t first variable) such that $\eta(t) H^{\Delta}(t, \eta(t), \xi(t))<0, \quad \forall \eta(t) \in \mathbb{R} \backsim\{0\}, \quad \forall \xi(t) \in \mathbb{R}, \quad \forall t \in \mathbb{T} ;$

$\left(\mathbf{U}_{\mathbf{5}}\right)\left|H^{\Delta}(t, \eta(t), \xi(t))\right| \geq p(t)|\eta(t)|, \quad \forall \eta(t) \in \mathbb{R} \backsim\{0\}, \quad \forall \xi(t) \in \mathbb{R}, t \in \mathbb{T} ;$

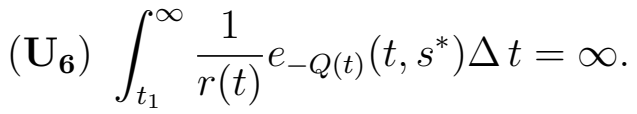

Now we establish two Lemmas, which will be used in the next section.

Lemma 1. Let $y(t)$ be an eventually positive solution of (4). Assume that $\left(U_{3}\right)-\left(U_{6}\right)$ and either $\left(U_{1}\right)$ or $\left(U_{2}\right)$ holds. Then there exists $s^{*}>t_{1}$ such that

$$
y(t)>0, \quad y^{\Delta}(t)>0 \quad \text { and } \quad\left(D\left(t, s^{*}\right) r(t) y^{\Delta}(t)\right)^{\Delta}<0 \quad \forall s^{*} \leq t .
$$

Proof. Since $y(t)$ is an eventually positive solution of (4). Take $t_{2} \in\left[t_{1}, \infty\right)_{\mathbb{T}}$ such that $y(t)>0$ on $\left[t_{2}, \infty\right)_{\mathbb{T}}$. In view of equation $(4)$ and $\left(U_{1}\right)-\left(U_{5}\right)$, we have

$$
\left(r(t) y^{\Delta}(t)\right)^{\Delta}+Q(t) r(t) y^{\Delta}(t) \leq-(p(t)-B(t)) r^{\sigma}(t) y(t)=-M(t) y(t)<0
$$

on $\left[t_{2}, \infty\right)_{\mathbb{T}}$. Now, by dividing equation (10) by a non-negative function $1-\mu(t) Q(t)$, we get

$$
\frac{\left(r(t) y^{\Delta}(t)\right)^{\Delta}}{1-\mu(t) Q(t)}+\frac{Q(t)}{1-\mu(t) Q(t)} r(t) y^{\Delta}(t)<0
$$

or

$$
\left(D\left(t, s^{*}\right) r(t) y^{\Delta}(t)\right)^{\Delta}<0 .
$$

Then $D\left(t, s^{*}\right) r(t) y^{\Delta}(t)$ is an eventually decreasing function and thus it is eventually of one sign, i.e. it is either eventually positive or eventually negative. We assert that $D\left(t, s^{*}\right) r(t) y^{\Delta}(t)$ is eventually non-negative. Suppose it is eventually negative, then there exist $t_{3} \geq t_{2}$ and a constant $K_{1}<0$ such that

$$
D\left(t, s^{*}\right) r(t) y^{\Delta}(t) \leq K_{1}<0 .
$$

Integrating the above relation from $t_{3}$ to $t$, we obtain

$$
\begin{aligned}
& y(t) \leq y\left(t_{3}\right)+K_{1} \int_{t_{3}}^{t} \frac{1}{r(x) D\left(x, s^{*}\right)} \Delta x= \\
& =y\left(t_{3}\right)+K_{1} \int_{t_{3}}^{t} \frac{1}{r(x)} e_{-Q(x)}\left(x, s^{*}\right) \Delta x \rightarrow-\infty \text { as } t \rightarrow \infty,
\end{aligned}
$$

which is a contradiction since $y(t)>0$ for all $t_{2} \leq t$. Hence, $D\left(t, s^{*}\right) r(t) y^{\Delta}(t)$ is eventually non-negative, i.e., $y^{\Delta}(t)$ is eventually positive function or there exists $s^{*} \in \mathbb{T}$ such that $y^{\Delta}(t)>0$ for all $t \in\left[s^{*}, \infty\right)_{\mathbb{T}}$.

Thus, we have

$$
y(t)>0, \quad y^{\Delta}(t)>0 \text { and }\left(D\left(t, s^{*}\right) r(t) y^{\Delta}(t)\right)^{\Delta}<0, \quad \forall s^{*} \leq t .
$$


Lemma 2. Let Lemma 1 hold. Futhermore, assume $r^{\Delta}(t) \geq 0$. Then

$$
\partial(t)<\frac{y(t)}{y^{\sigma}(t)}<1
$$

where $\check{\partial}(t)=\frac{t-s^{*}}{t-s^{*}+\mu(t)}$.

Proof. From (9), we obtain

$$
y(t)>0, \quad y^{\Delta}(t)>0, \quad y^{\Delta \Delta}(t)<0 .
$$

Also from (12), we have

$$
y(t)=y(t)-y\left(s^{*}\right)=\int_{s^{*}}^{t} y^{\Delta}(\eta) \Delta \eta>y^{\Delta}(t)\left(t-s^{*}\right) .
$$

From (13) and $y^{\sigma}(t)=y(t)+\mu(t) y^{\Delta}(t)$, we get

$$
\partial(t) \leq \frac{y(t)}{y^{\sigma}(t)} \leq 1
$$

\section{Main Results}

In this section, we give some new oscillation criteria for equation (4).

Theorem 5. Assume that $\left(U_{3}\right)-\left(U_{6}\right)$ and either $\left(U_{1}\right)$ or $\left(U_{2}\right)$ hold. Furthermore, there exists $\delta(t) \in{ }^{+} \mathbb{C}_{r d}^{\Delta}(\mathbb{T})$ (not necessary $r d$-continuity of $\delta^{\Delta}$ ) such that for all sufficiently large $s^{*}$,

$$
\limsup _{t \rightarrow \infty} \int_{s^{*}}^{t}\left[M(\xi) \delta(\xi)-\frac{A_{1}^{2}(\xi)}{4 A_{2}(\xi)}\right] \Delta \xi=\infty
$$

or

$$
\limsup _{t \rightarrow \infty} \int_{s^{*}}^{t}\left[M(\xi) \delta(\xi)-\frac{r(\xi)\left(\delta^{\Delta}(\xi)-\delta(\xi) Q(\xi)\right)^{2}}{4 \delta(\xi)}\right] \Delta \xi=\infty .
$$

Then, every solution of equation (4) is oscillatory.

Proof. Suppose to the contrary that $y(t)$ is a non-oscillatory solution of equation (4). We may assume without loss of generality that $y(t)$ is eventually positive. By Lemma 1, we have

$$
y(t)>0, \quad y^{\Delta}(t)>0 \text { and }\left(D\left(t, s^{*}\right) r(t) y^{\Delta}(t)\right)^{\Delta}<0 \quad \forall s^{*} \leq t .
$$

Now define a function $w(t)$ by the Riccati substitution

$$
w(t)=\delta(t) \frac{r(t) y^{\Delta}(t)}{y(t)}, \quad t \geq s^{*} \geq t_{1} .
$$

Then, $w(t)>0$ and

$$
w^{\Delta}(t)=\left(\delta(t) \frac{r(t) y^{\Delta}(t)}{y(t)}\right)^{\Delta}=\left(r(t) y^{\Delta}(t)\right)^{\sigma}\left(\frac{\delta(t)}{y(t)}\right)^{\Delta}+\left(r(t) y^{\Delta}(t)\right)^{\Delta}\left(\frac{\delta(t)}{y(t)}\right) .
$$

From (17), we have

$$
w^{\Delta}(t)=\frac{w^{\sigma}(t)}{\delta^{\sigma}(t)}\left(\delta^{\Delta}(t)-\frac{\delta(t) y^{\Delta}(t)}{y(t)}\right)+\left(r(t) y^{\Delta}(t)\right)^{\Delta}\left(\frac{\delta(t)}{y(t)}\right)
$$


From (10) and (17), we obtain

$$
\begin{aligned}
& w^{\Delta}(t) \leq \frac{w^{\sigma}(t)}{\delta^{\sigma}(t)}\left(\delta^{\Delta}(t)-\frac{\delta(t) y^{\Delta}(t)}{y(t)}\right)-\left(M(t) y(t)+Q(t)\left(r(t) y^{\Delta}(t)\right)\right)\left(\frac{\delta(t)}{y(t)}\right)= \\
& =-M(t) \delta(t)-Q(t) w(t)+\frac{\delta^{\Delta}(t) w^{\sigma}(t)}{\delta^{\sigma}(t)}-\frac{w^{\sigma}(t) w(t)}{r(t) \delta^{\sigma}(t)} .
\end{aligned}
$$

Since $D\left(t, s^{*}\right) r(t) y^{\Delta}(t)$ is eventually decreasing function, then

$$
D^{\sigma}\left(t, s^{*}\right)\left(r(t) y^{\Delta}(t)\right)^{\sigma} \leq D\left(t, s^{*}\right) r(t) y^{\Delta}(t), \quad \text { as } t \leq \sigma(t) .
$$

From (17), we obtain

$$
w(t) \geq \frac{D^{\sigma}\left(t, s^{*}\right) y^{\sigma}(t) \delta(t) w^{\sigma}(t)}{D\left(t, s^{*}\right) y(t) \delta^{\sigma}(t)} .
$$

Also, since $y(t)$ is eventually increasing function, then we get

$$
y(t) \leq y^{\sigma}(t)
$$

From (19) and (20), we have

$$
w(t) \geq \frac{D^{\sigma}\left(t, s^{*}\right) \delta(t) w^{\sigma}(t)}{D\left(t, s^{*}\right) \delta^{\sigma}(t)}
$$

or

$$
-Q(t) w(t) \leq-\frac{D^{\sigma}\left(t, s^{*}\right) \delta(t) w^{\sigma}(t)}{D\left(t, s^{*}\right) \delta^{\sigma}(t)} Q(t)
$$

From (19) and (21), we obtain

$$
\begin{aligned}
& w^{\Delta}(t) \leq-M(t) \delta(t)-\frac{D^{\sigma}\left(t, s^{*}\right) \delta(t) w^{\sigma}(t)}{D\left(t, s^{*}\right) \delta^{\sigma}(t)} Q(t)+\frac{\delta^{\Delta}(t) w^{\sigma}(t)}{\delta^{\sigma}(t)}- \\
& -\frac{\left(w^{\sigma}(t)\right)^{2} D^{\sigma}\left(t, s^{*}\right) \delta(t)}{r(t) D\left(t, s^{*}\right)\left(\delta^{\sigma}(t)\right)^{2}}=-M(t) \delta(t)+A_{1}(t) w^{\sigma}(t)-\left(w^{\sigma}(t)\right)^{2} A_{2}(t)= \\
& =-M(t) \delta(t)-\left[w^{\sigma}(t) \sqrt{A_{2}(t)}-\frac{A_{1}(t)}{2 \sqrt{A_{2}(t)}}\right]^{2}+\frac{A_{1}^{2}(t)}{4 A_{2}(t)}
\end{aligned}
$$

Hence

$$
w^{\Delta}(t) \leq-\left[M(t) \delta(t)-\frac{A_{1}^{2}(t)}{4 A_{2}(t)}\right]
$$

Integrating (23) from $s^{*}$ to $t$, we obtain

$$
\int_{s^{*}}^{t}\left[M(\xi) \delta(\xi)-\frac{A_{1}^{2}(\xi)}{4 A_{2}(\xi)}\right] \Delta \xi \leq w\left(s^{*}\right)-w(t) \leq w\left(s^{*}\right)<\infty,
$$

for all large $t$. Which is a contradiction because of relation (15). Hence, the proof is complete due to asumption (15). 
Now we prove the result using (16). From equations (19), (20) and $D^{\sigma}\left(t, s^{*}\right) \geq D\left(t, s^{*}\right)$, we obtain

$$
w(t) \geq \frac{\delta(t) w^{\sigma}(t)}{\delta^{\sigma}(t)}
$$

Similarly, from (23) and (25), we obtain

$$
\left[M(\xi) \delta(\xi)-\frac{r(\xi)\left(\delta^{\Delta}(\xi)-\delta(\xi) Q(\xi)\right)^{2}}{4 \delta(\xi)}\right] \leq-w^{\Delta}(t)
$$

Integrating equation (16) from $s^{*}$ to $t$, we get

$$
\int_{s^{*}}^{t}\left[M(\xi) \delta(\xi)-\frac{r(\xi)\left(\delta^{\Delta}(\xi)-\delta(\xi) Q(\xi)\right)^{2}}{4 \delta(\xi)}\right] \Delta \xi \leq w\left(s^{*}\right)-w(t) \leq w\left(s^{*}\right)<\infty
$$

for sufficiently large $t$, which is a contradiction as (16) holds. Hence, the proof is complete due to assumption (16). Thus, the whole proof is complete.

As an immediate consequence of Theorem (5), we have the following corollaries for different values of $\delta(t)$.

For $\delta(t)=$ constant (say, $C>0), t \geq t_{1}$, we have the following result.

Corollary 1. Assume that $\left(U_{3}\right)-\left(U_{6}\right)$ and either $\left(U_{1}\right)$ or $\left(U_{2}\right)$ hold. Furthermore, assume

$$
\limsup _{t \rightarrow \infty} \int_{s^{*}}^{t}\left[M(\xi)-\frac{r(\xi) D^{\sigma}\left(\xi, s^{*}\right) Q^{2}(\xi)}{4 D\left(\xi, s^{*}\right)}\right] \Delta \xi=\infty
$$

or

$$
\limsup _{t \rightarrow \infty} \int_{s^{*}}^{t}\left[M(\xi)-\frac{r(\xi) Q^{2}(\xi)}{4}\right] \Delta \xi=\infty .
$$

Then, every solution of equation (4) is oscillatory.

For $\delta(t)=t^{2}$, we have following result.

Corollary 2. Assume that $\left(U_{3}\right)-\left(U_{6}\right)$ and either $\left(U_{1}\right)$ or $\left(U_{2}\right)$ hold. Furthermore, assume

$$
\limsup _{t \rightarrow \infty} \int_{s^{*}}^{t}\left[M(\xi) \xi^{2}-\frac{\left(\frac{(\xi+\sigma(\xi))}{(\sigma(\xi))^{2}}-\frac{D^{\sigma}\left(\xi, s^{*}\right) \xi^{2}}{D\left(\xi, s^{*}\right)(\sigma(\xi))^{2}} Q(\xi)\right)^{2}}{\left(\frac{4 D^{\sigma}\left(\xi, s^{*}\right) \xi^{2}}{r(\xi)(\sigma(\xi))^{4} D\left(\xi, s^{*}\right)}\right)}\right] \Delta \xi=\infty
$$

or

$$
\limsup _{t \rightarrow \infty} \int_{s^{*}}^{t}\left[M(\xi) \xi^{2}-\frac{r(\xi)\left(\xi+\sigma(\xi)-\xi^{2} Q(\xi)\right)^{2}}{4 \xi^{2}}\right] \Delta \xi=\infty .
$$

Then, every solution of equation (4) is oscillatory.

We add one more condition, i.e. $r^{\Delta}(t) \geq 0$ or $r(t) \in{ }^{+} \mathbb{C}_{r d}^{\Delta+}(\mathbb{T})$ in Theorem (5) to obtain some new oscillation criteria of equation (4). 
Theorem 6. Assume that $\left(U_{3}\right)-\left(U_{6}\right)$ and either $\left(U_{1}\right)$ or $\left(U_{2}\right)$ hold. Moreover, $r^{\Delta}(t) \geq 0$ or $r(t) \in{ }^{+} \mathbb{C}_{r d}^{\Delta+}(\mathbb{T})$ and there exists $\delta(t) \in{ }^{+} \mathbb{C}_{r d}^{\Delta}(\mathbb{T})$ (not necessary rd-continuity of $\delta^{\Delta}$ ) such that for all sufficiently large $s^{*}$,

$$
\limsup _{t \rightarrow \infty} \int_{s^{*}}^{t}\left[M(\xi) \delta^{\sigma}(\xi) ð(\xi)-\frac{B_{1}^{2}(\xi)}{4 B_{2}(\xi)}\right] \Delta \xi=\infty .
$$

Then, every solution of equation (4) is oscillatory.

Proof. In view of Theorem (5) and equation (17), we obtain

$$
w^{\Delta}(t)=\delta^{\Delta}(t)\left(\frac{r(t) y^{\Delta}(t)}{y(t)}\right)+\delta^{\sigma}(t)\left(\frac{r(t) y^{\Delta}(t)}{y(t)}\right)^{\Delta} .
$$

By using equation (10), Lemma 2 and after manipulation, we obtain

$$
w^{\Delta}(t) \leq-M(t) \delta^{\sigma}(t) \partial(t)+B_{1}(t) w(t)-B_{2}(t) w^{2}(t),
$$

which is equivalent to

$$
w^{\Delta}(t) \leq-M(t) \delta^{\sigma}(t) ð(t)-\left[w(t) \sqrt{B_{2}(t)}-\frac{B_{1}(t)}{2 \sqrt{B_{2}(t)}}\right]^{2}+\frac{B_{1}^{2}(t)}{4 B_{2}(t)} .
$$

Integrating the above equation from $s^{*}$ to $t$, we obtain

$$
\int_{s^{*}}^{t}\left[M(\xi) \delta^{\sigma}(\xi) \partial(\xi)-\frac{B_{1}^{2}(\xi)}{4 B_{2}(\xi)}\right] \Delta \xi \leq w\left(s^{*}\right)-w(t) \leq w\left(s^{*}\right)<\infty,
$$

for all large $t$, which is a contradiction due to (28). Hence, the proof is complete.

Now as a special case if $\delta(t)$ is positive constant, we obtain a result:

Corollary 3. Assume that $\left(U_{3}\right)-\left(U_{6}\right)$ and either $\left(U_{1}\right)$ or $\left(U_{2}\right)$ hold. Moreover, $r^{\Delta}(t) \geq 0$ or $r(t) \in{ }^{+} \mathbb{C}_{r d}^{\Delta+}(\mathbb{T})$ and

$$
\limsup _{t \rightarrow \infty} \int_{s^{*}}^{t} \precsim(t)\left[M(\xi)-\frac{r(\xi) Q^{2}(\xi)}{4}\right] \Delta \xi=\infty .
$$

Then, every solution of equation (4) is oscillatory.

Now we establish Kamenev-type oscillation criteria for (4). We need the following result of [16] $\left((t-s)^{m}\right)^{\Delta_{s}} \leq-m(t-\sigma(s))^{m-1} \leq 0$ for $m>1$ and $\sigma(s) \leq t$ to establish our results.

Theorem 7. Assume that $\left(U_{3}\right)-\left(U_{6}\right)$ and either $\left(U_{1}\right)$ or $\left(U_{2}\right)$ holds. Furthermore, there exists $\delta(t) \in+\mathbb{C}_{r d}^{\Delta}(\mathbb{T})$ (not necessary rd-continuity of $\delta^{\Delta}$ ) such that for $\aleph>1$ and for all sufficiently large $s^{*}$,

$$
\limsup _{t \rightarrow \infty} \frac{1}{t^{\aleph}} \int_{s^{*}}^{t}(t-\xi)^{\aleph}\left[M(\xi) \delta(\xi)-\frac{A_{1}^{2}(\xi)}{4 A_{2}(\xi)}\right] \Delta \xi=\infty
$$

or 


$$
\limsup _{t \rightarrow \infty} \frac{1}{t^{\aleph}} \int_{s^{*}}^{t}(t-\xi)^{\aleph}\left[M(\xi) \delta(\xi)-\frac{r(\xi)\left(\delta^{\Delta}(\xi)-\delta(\xi) Q(\xi)\right)^{2}}{4 \delta(\xi)}\right] \Delta \xi=\infty .
$$

Then, every solution of (4) is oscillatory.

Proof. In view of Theorem 5, from (23), we have

$$
w^{\Delta}(t) \leq-\left[M(t) \delta(t)-\frac{A_{1}^{2}(t)}{4 A_{2}(t)}\right]
$$

Thus

$$
\int_{s^{*}}^{t}(t-\xi)^{\aleph} w^{\Delta}(\xi) \Delta \xi \leq-\int_{s^{*}}^{t}(t-\xi)^{\aleph}\left[M(\xi) \delta(\xi)-\frac{A_{1}^{2}(\xi)}{4 A_{2}(\xi)}\right] \Delta \xi
$$

We know

$$
\int_{s^{*}}^{t}(t-\xi)^{\aleph} w^{\Delta}(\xi) \Delta \xi=-\left(t-s^{*}\right)^{\aleph} w\left(s^{*}\right)-\int_{s^{*}}^{t}\left((t-\xi)^{\aleph}\right)^{\Delta_{\xi}} w(\sigma(\xi)) \Delta \xi,
$$

and using the remark (3.3) in [16], we have

$$
\left((t-\xi)^{\aleph}\right)^{\Delta_{\xi}} \leq-\aleph(t-\sigma(\xi))^{\aleph-1} \leq 0, \sigma(\xi) \leq t \text { and } \aleph>1 .
$$

From equations (33), (34) and (35), we obtain

$$
\begin{aligned}
& -\int_{s^{*}}^{t}(t-\xi)^{\aleph}\left[M(\xi) \delta(\xi)-\frac{A_{1}^{2}(\xi)}{4 A_{2}(\xi)}\right] \Delta \xi \geq \int_{s^{*}}^{t}(t-\xi)^{\aleph} w^{\Delta}(\xi) \Delta \xi=-\left(t-s^{*}\right)^{\aleph} w\left(s^{*}\right)- \\
& -\int_{s^{*}}^{t}\left((t-\xi)^{\aleph}\right)^{\Delta_{\xi}} w(\sigma(\xi)) \Delta \xi \geq-\left(t-s^{*}\right)^{\aleph} w\left(s^{*}\right),
\end{aligned}
$$

or

Thus

$$
\int_{s^{*}}^{t}(t-\xi)^{\aleph}\left[M(\xi) \delta(\xi)-\frac{A_{1}^{2}(\xi)}{4 A_{2}(\xi)}\right] \Delta \xi \leq\left(t-s^{*}\right)^{\aleph} w\left(s^{*}\right)
$$

$$
\limsup _{t \rightarrow \infty} \frac{1}{t^{\aleph}} \int_{s^{*}}^{t}(t-\xi)^{\aleph}\left[M(\xi) \delta(\xi)-\frac{A_{1}^{2}(\xi)}{4 A_{2}(\xi)}\right] \Delta \xi \leq \limsup _{t \rightarrow \infty}\left(1-\frac{s^{*}}{t}\right)^{\aleph} w\left(s^{*}\right)<\infty .
$$

Which contradicts assumption (31). To prove the result using (32), we apply the above process for equation (26), then we get a contradiction since we have (32). Hence, the proof is complete.

Theorem 8. Assume that $\left(U_{3}\right)-\left(U_{6}\right)$ and either $\left(U_{1}\right)$ or $\left(U_{2}\right)$ holds. Moreover, either $r^{\Delta}(t) \geq 0$ or $r(t) \in{ }^{+} \mathbb{C}_{r d}^{\Delta+}(\mathbb{T})$ and there exists $\delta(t) \in{ }^{+} \mathbb{C}_{r d}^{\Delta}(\mathbb{T})$ (not necessary rd-continuity of $\delta^{\Delta}$ ) such that for $\aleph>1$ and for all sufficiently large $s^{*}$,

$$
\limsup _{t \rightarrow \infty} \frac{1}{t^{\aleph}} \int_{s^{*}}^{t}(t-\xi)^{\aleph}\left[M(\xi) \delta^{\sigma}(\xi) ð(\xi)-\frac{B_{1}^{2}(\xi)}{4 B_{2}(\xi)}\right] \Delta \xi=\infty .
$$

Then, every solution of equation (4) is oscillatory.

Proof of the above Theorem is same as proof of Theorem 8. 
Remark 2. The result of Theorem 8 and Theorem 9 holds for any $\delta(t) \in+\mathbb{C}_{r d}^{\Delta}(\mathbb{T})$ (not necessary rd-continuity of $\delta^{\Delta}$ ). Thus, by these Theorems, we can immediately obtain corollaries with different choice of $\delta(t)$.

Let us consider a second order non-linear dynamic equation with deviating argument on an arbitrary time scale $\mathbb{T}$ :

$$
y^{\Delta \Delta}(t)+\beta y^{\Delta}(t)=B(t) y(t)+\mathcal{W}^{\Delta}\left(t, y(t), y\left(w_{1}(t, y(t))\right)\right)
$$

where $\beta \geq 0, w_{1}(t, y(t))=b_{1}\left(t, y\left(b_{2}\left(t, \cdots, y\left(b_{m_{0}}(t, y(t))\right) \cdots\right)\right)\right), B$ is a function of $t$, and the forcing terms $\mathcal{W}$ and $b_{i}, i=1,2, \cdots, m_{0}$.

Remark 3. If we replace the integro forcing term $H\left(t, y(t), \int_{-\infty}^{t} \mathcal{J}(t-s) \mathcal{H}(s, y(s)) \Delta s\right)$ (in 3) by the deviating argument $\mathcal{W}\left(t, y(t), y\left(w_{1}(t, y(t))\right)\right.$ ) (in 37), then in the similar manner we can obtain oscillation criteria of (37). Thus, all the above Theorems and corollaries can be achieved for the second order dynamic equation (37), i.e., all the results will remain the same for it.

\section{References}

1. Agarwal R.P. Difference Equations and Inequalities. N.Y., Marcel Dekker, 1992.

2. Agarwal R.P., Wong P.J.Y. Advanced Topics in Difference Equations. Dordrecht, Springer Netherlands, 1997. DOI: 10.1007/978-94-015-8899-7

3. Jurang Y. Oscillation Theorems for Second Order Linear Differential Equations with Damping. Proceeding of American Mathematical Society, 1986, vol. 98, no. 2, pp. 276-282.

4. Hilger S. Analysis on Measure Chains - A Unified Approach to Continuous and Discrete Calculus. Results in Mathematics, 1990, vol. 18, issue 1-2, pp. 18-56. DOI: $10.1007 / \mathrm{BF} 03323153$

5. Bohner M., Peterson A. Dynamic Equations on Time Scales: An Introduction with Applications. Boston, Birkhauser, 2001. DOI: 10.1007/978-1-4612-0201-1

6. Bohner M., Peterson A. Advances in Dynamic Equations on Time Scales. Boston, Birkhauser, 2003. DOI: $10.1007 / 978-0-8176-8230-9$

7. Atici F.M., Biles D.C., Lebedinsky A. An Application of Time Scales to Economics. Mathematical and Computer Modelling, 2006, vol. 43, issues 7-8, pp. 718-726. DOI: $10.1016 / \mathrm{j} . \mathrm{mcm} .2005 .08 .014$

8. Christiansen F.B., Fenchel T.M. Theories of Populations in Biological Communities. Lecture Notes in Ecological Studies, vol. 20, Berlin, Springer-Verlag, 1977, pp. 1-36.

9. Agarwal R. P., O'regan D., Saker S. H. Oscillation Criteria for Nonlinear Perturbed Dynamic Equations of Second-Order on Time Scales. Journal of Applied Mathematics and Computing, 2006, vol. 20, issues 1-2, pp. 133-147. DOI: $10.1007 /$ BF02831928

10. Bohner M., Saker S. H. Oscillation of Second Order Nonlinear Dynamic Equations on Time Scales. Rocky Mountain Journal of Mathematics, 2004, vol. 34, no. 4, pp. 1239-1254.

11. Chen W., Han Z., Sun S., Li T. Oscillation Behavior of a Class of Second-Order Dynamic Equations with Damping on Time Scales. Discrete Dynamic in Nature and Society, 2010, Article ID 907130. 15 p.

12. Da-Xue C., Guang-Hui L. Oscillation Criteria for Non-Linear Second-Order Damped Delay. Dynamic Equations on Time Scales, World Academy of Science, Engineering and Technology, International Journal of Mathematical, Computational, Physical, Electrical and Computer Engineering, 2010, vol. 4, no. 1, pp. 185-192. 
13. Erbe L., Peterson A., Saker S.H. Oscillation Criteria for Second-Order Non-Linear Dynamic Equations on Time Scales. Journal of London Mathematical Society, 2003, vol. 67, issue 3, pp. 701-714. DOI: $10.1112 /$ S0024610703004228

14. Saker S. H., Agarwal R. P., O'Regan D. Oscillation of Second-Order Damped Dynamic Equations on Time Scales. Journal of Mathematical Analysis and Applications, 2007, vol. 330, issue 2, pp. 1317-1337. DOI: 10.1016/j.jmaa.2006.06.103

15. Erbe L., Hassan T.S., Peterson A. Oscillation Criteria for Non-Linear Damped Dynamic Equations on Time Scales. Applied Mathematics and Computation, 2008, vol. 203, issue 1, pp. 343-357. DOI: $10.1016 /$ j.amc.2008.04.038

16. Saker S. H. Oscillation of Second-Order Non-Linear Neutral Delay Dynamic Equations on Time Scales. Journal of Computational and Applied Mathematics, 2006, vol. 187, issue 2, pp. 123-141. DOI: 10.1016/j.cam.2005.03.039

17. Agacik Z. On Oscillation and Non-Oscillation of Second-Order Dynamic Equations. Applied Mathematics Letters, 2009, vol. 22, issue 1, pp. 136-141. DOI: 10.1016/j.aml.2008.03.003

18. Guseinov G. Sh., Kaymakcalan B. On a Disconjugacy Criterion for Second Order Dynamic Equations on Time Scales. Journal of Computational Applied Mathematics, 2002, vol. 141, issues 1-2, pp. 187-196. DOI: 10.1016/S0377-0427(01)00445-9

Received November 11, 2016

УДК 517.9

DOI: $10.14529 / \mathrm{mmp} 170103$

\title{
КРИТЕРИИ КОЛЕБАНИЙ НЕЛИНЕЙНЫХ ДИНАМИЧЕСКИХ УРАВНЕНИЙ ВТОРОГО ПОРЯДКА С ИНТЕГРАЛЬНОЙ СОСТАВЛЯЮЩЕЙ НА ВРЕМЕННЫХ МАСШТАБАХ
}

\author{
II.C. Неги, С. Аббас, М. Малик
}

В статье рассматриваются колебательные свойства нелинейного динамического уравнения второго порядка с интегральной составляющей на произвольном промежутке времени. При помощи введения оператора сдвига исходное динамическое уравнение редуцируется к альтернативному уравнению. Для изучения колебаний мы представим некоторые важные леммы и будем использовать обобщенное преобразование Риккати, которое переводит динамическое уравнение второго порядка в динамическое уравнение первого порядке на произвольном промежутке времени. Полученные результаты также гарантируют, что решение исходного уравнения осциллирует. Кроме того, мы устанавливаем критерий колебаний Каменева для нашей системы. В итоге, мы рассмотрим динамическое уравнение второго порядка на временных масштабах с отклоняющимся аргументом и сравним его с результатом, который дает достаточные условия его колебания.

Ключевые слова: масштаб времени; динамические уравнения; преобразование Риккати; колебание.

Шекхар Сингх Неги, Школа фундаментальных наук, Индийский институт технологий в Манди (г. Манди, Индия), sssshekhar.singh17@gmail.com.

Сайед Аббас, Школа фундаментальных наук, Индийский институт технологий в Манди, (г. Манди, Индия), sabbas.iitk@gmail.com.

Муслим Малик, Школа фундаментальных наук, Индийский институт технологий в Манди, (г. Манди, Индия), malikiisc@gmail.com.

Поступила в редакиию 11 ноября 2016 г. 